\title{
The Simulation Analysis on Mechanical Properties of Plastic Cantilever Buckle
}

\author{
Huifen Lai \\ College of Mechanical and Electrical Engineering, China jiliang University, Hangzhou, Zhejiang \\ 310018, China \\ huifenlai@163.com
}

Keywords: Buckle design, Finite element analysis, Pro/E

Abstract. The study focused on plastic cantilever buckle. Combing with actual work and using plastic injection molding theory, the characteristics of conventional cantilever buckle model are summed up. The classical analysis on cantilever buckle the shape parameters and the mechanical assembly performance are reduced. The finite element simulation module mechanism on stress and strain of cantilever buckle are explored by using Pro/E 5.0 software to obtain the correction coefficient of the cantilever buckle and to obtain some optimization design measures. Since the analysis calculations are based on simplified model and certain assumptions, so the results of the analysis are not exactly, but it can satisfy the precision requirement of product design.

\section{Introduction}

Using plastic card hook the elastic deformation, the plastic buckle realize quick installation and disassembly of a connection between the two parts. In today's life, plastic buckle connecting piece can be seen everywhere, we can see it in the shell products, toys, bags, home appliances and other plastic products, the plastic buckle connection has been widely used. More and more people start to focus on plastic buckle connection, and carries on the research and design.

The plastic cantilever clasp is mainly composed of two types of components, respectively, the deflection element providing the elastic deformation in the assembly or disassembly and the holder to maintain the strength. As shown in Fig. 1.below.

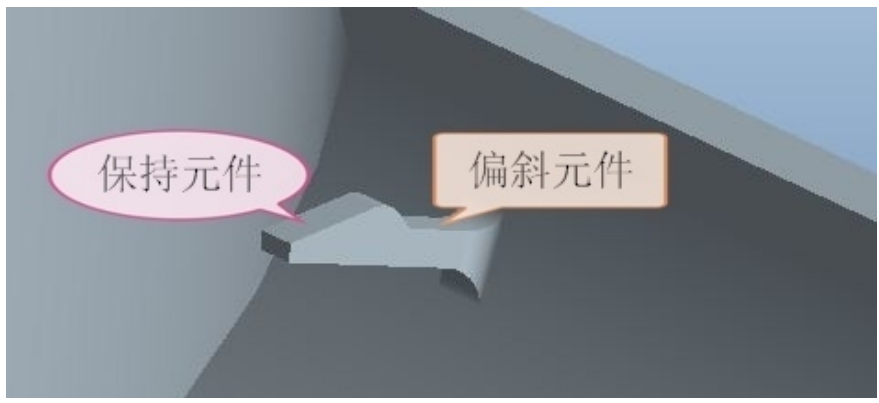

Fig. 1 main component of the plastic buckle

\section{The calculation and analysis of the relevant parameters on plastic buckle design}

\section{Length of cantilever beam}

The length of cantilever beam ( $\mathrm{Lb}$ ) is the length of skew component. According to beam theory, $\mathrm{Lb}$ at least equal to 5 times the thickness of the wall. If the length is less than 5 times the thickness of the wall, the beam theory is still used to analyze and calculate, the results will be distorted. 


\section{Angle of insertion}

The size of the angle of the insertion will directly affect the size of the assembly force, The bigger the insertion angle, the greater the assembly force. So the insertion angle should be reduced as much as possible to reduce the assembly force, under the condition that the length of the beam is allowed, By means of the data, it can be found that the reasonable insertion angle is between 25 degree and 35 degree. An angle greater than or equal to 45 degrees will make the assembly difficult.

\section{Clip root width on plastic buckle}

The change of buckle width is small from the cantilever root to the retaining surface, only a small draft angle. At this time, the buckle width does not affect the maximum assembly strain, but it affects the assembly force, the output force and the hold strength. From beam theory, the width of the cantilever should be less than the length of the beam 1/2, The closer the buckle width is close to the length of the beam, the greater the error when applying the beam theory.

\section{Determination of assembling clearance on}

Because there is always a certain deviation in the assembly process of the cantilever buckle, the contact surface of the plastic buckle and the mating part must be designed to have a certain gap, In addition to the plastic products in the production process, there will be a certain error, due to mold processing, injection molding and other reasons, The dimensions and tolerances of the plastic products are directly related to the shrinkage rate of the plastic products, So we should to meet these needs. in the design of the buckle gaps.

According to the national plastic parts shape and position tolerance standard to select the appropriate size tolerance, under normal circumstances, in order to facilitate the memory can be approximated by the following formula to take the design formula: $G=$ size A GB tolerance $\mathrm{x} 2+$ $0.1(\mathrm{~mm})$.

\section{The simulation analysis of mechanical properties on plastic cantilever buckle}

Set up the assembly model of the outer panel and the inner panel with Pro/E5.0 software

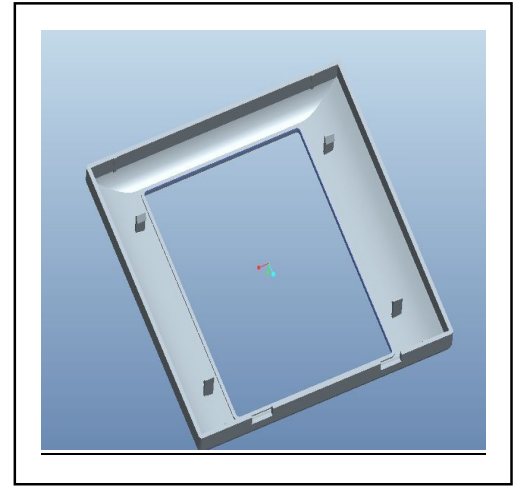

Fig.2 the outer panel

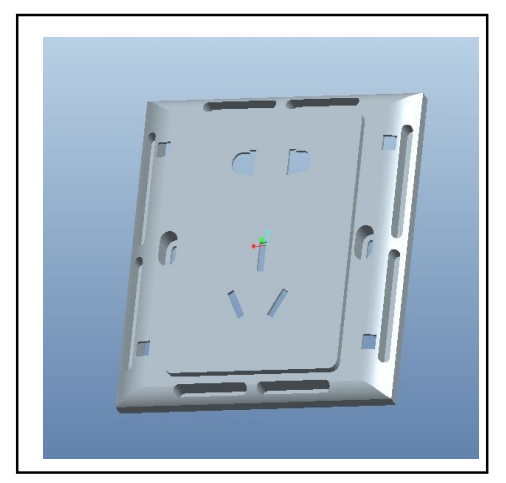

Fig.3 the outer panel

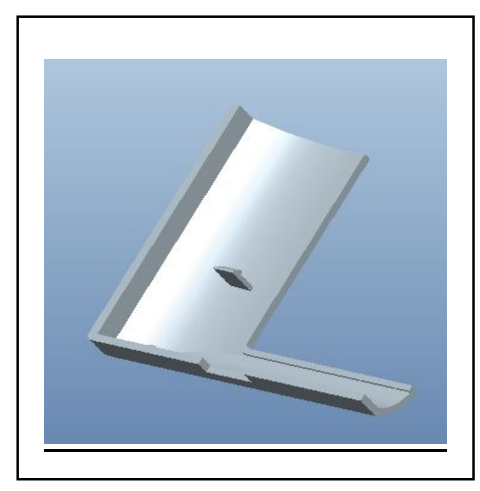

Fig.4 1/4 part model

Taking into account the symmetry of the model and the time of solution, in the standard module, the whole model is cut into the $1 / 4$ model, as shown in the figure 4 .

The grid is divided on the panel, and the mesh quality is controlled by dimension the size of the grid, the refine the mesh is used. between the buckle and the slot contact area.

To establish the fixed constraints of the inner panel, the two shear planes of the selected $1 / 4$ model are used as constraints, material: density $0.95 \mathrm{~g} / \mathrm{cm} 3$, Elastic modulus1200MPa, Poisson ratio 0.41 . To establish the buckle structure based on the fourth strength theory, In the process of 
clamping force, the stress along buckle cantilever length direction is shown below figures.

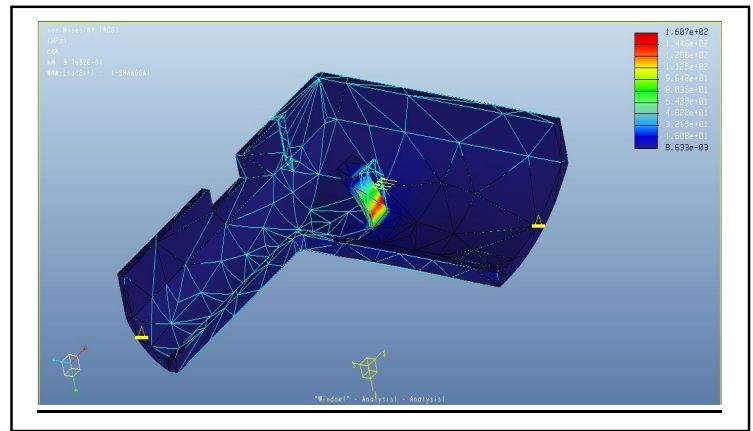

Fig.5 Stress fringe display

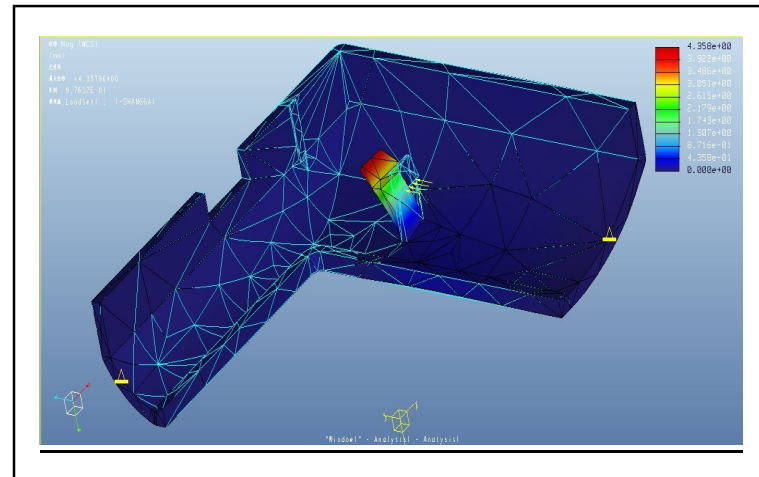

Fig. 7 displacement fringe display

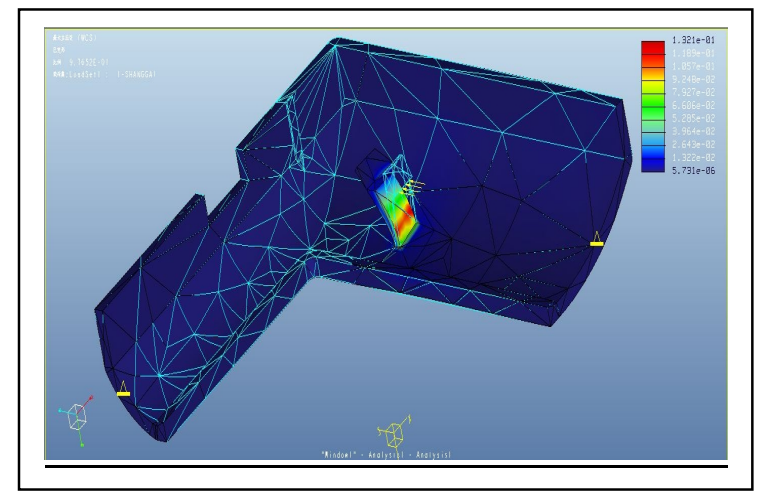

Fig. 9 stress fringe display

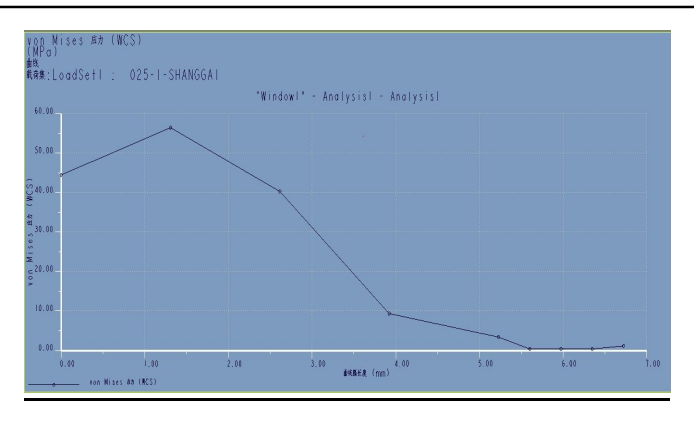

Fig.6 Stress-length graphic display

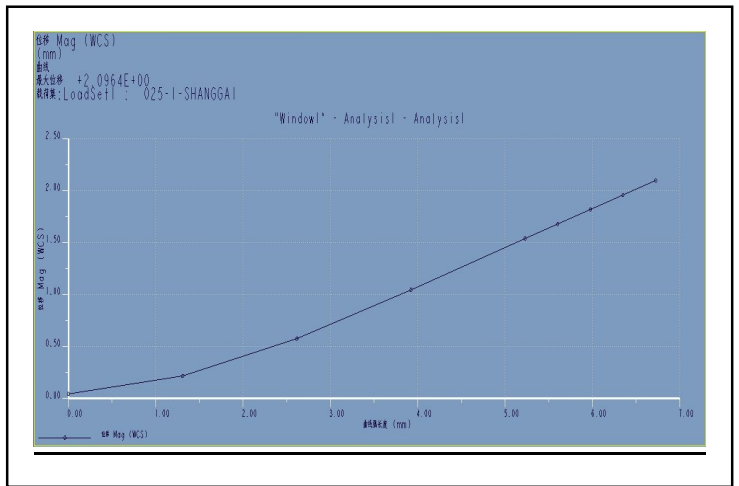

Fig. 8 displacement - length graphic display

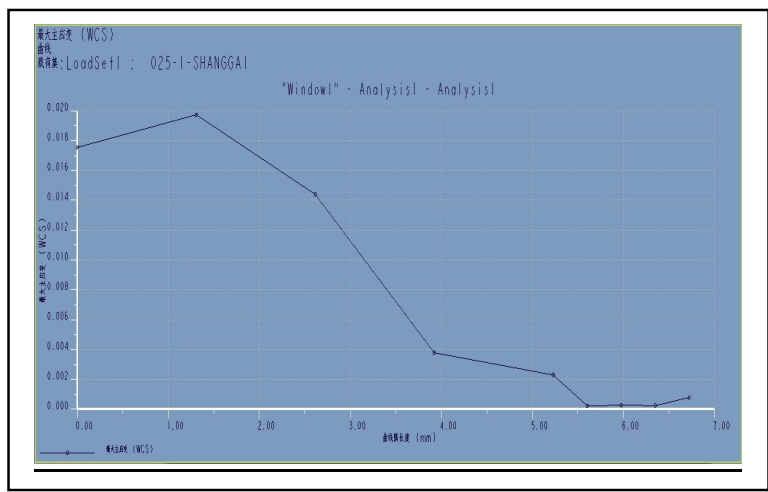

Fig.10 strain length graphic display

Can be seen from the chart, the maximum strain of the buckle in the course of stress is much smaller than the maximum bending strain of the raw material $6 \%$, So the buckle in the stress process does not appear the phenomenon of structural damage. The maximum stress occurs at the moment of the maximum bending moment of the buckle, while the maximum deviation occurred in the clamping insert surface and the clamping slot guide most intense, the moment before the buckle is about to enter the slot. The maximum stress area (red color) occurs at the junction of the face near the buckle. The maximum equivalent stress of the buckle is less than the flexural strength of material. Buckle can meet the strength requirements.

\section{Conclusion}

At present, the domestic engineers generally design the plastic cantilever buckle with experience method, and then proofing verification. This efficiency is relatively low. The purpose of this paper is mainly to design of a reasonable and reliable cantilever plastic buckle for a plastic products by comparing the traditional analysis method with the finite element analysis method. 
By using the finite element analysis method to obtain the stress, strain and other mechanical parameters in the process of buckle assembly, Analysis of the data compared with the original data to final determination the rationality of the design on the plastic cantilever buckle.

\section{References}

[1] Chen Yan, Research on the design of plastic buckle and the development of CAD Application module. Master's degree thesis of Jiangnan University.

[2] Chen Yu, Hu Lanfang, Song Jianhua, The finite element analysis and structural improvement of the assembly of the snap fastener [J]. Technology innovation. 2013 (39).

[3] Lin Quan, He Liang, Wu Xiongfei, Buckle plastic parts analysis and its innovative molding die design.Plastic science and technology. 2013 (07). 